\title{
On oscillatory pattern of malaria dynamics in a population with temporary immunity
}

\author{
J. TUMWIINE $\dagger$, J. Y. T. MUGISHA $* *$ and L. S. LUBOOBI
}

$\dagger$ Department of Mathematics, Mbarara University, P.O. Box 1410, Mbarara, Uganda \$Department of Mathematics, Makerere University, P.O. Box 7062, Kampala, Uganda

(Received 2 October 2006; revised 1 February 2007; in final form 20 June 2007)

\begin{abstract}
We use a model to study the dynamics of malaria in the human and mosquito population to explain the stability patterns of malaria. The model results show that the disease-free equilibrium is globally asymptotically stable and occurs whenever the basic reproduction number, $R_{0}$ is less than unity. We also note that when $R_{0}>1$, the disease-free equilibrium is unstable and the endemic equilibrium is stable. Numerical simulations show that recoveries and temporary immunity keep the populations at oscillation patterns and eventually converge to a steady state.
\end{abstract}

Keywords: Malaria; Oscillatory pattern; Temporary immunity; Endemic stability

\section{Introduction}

The development of malaria parasites in a human host begins in the liver cells where the malaria parasites undergo asexual multiplication to produce merozoites that are eventually released into the blood stream to invade red blood cells. The infected red blood cells burst after 2-3 days to release merozoites and gametocytes into the blood stream. This is associated with the clinical symptoms of the disease. Mosquitoes become infected when they feed and ingest human blood that contains mature gametocytes. The gametocytes develop into male and female gametes that fertilize to become zygotes in the mid-gut wall of the mosquito. The zygote elongates to become ookinete and penetrates the mid-gut epithelium that later develop and ultimately produce sporozoites which become infective when they migrate to the salivary glands. The prevalence of the Plasmodium infections in humans mainly depends on the distribution of Anopheles mosquitoes.

Protective immunity against malaria can be thought of in different categories. There are two types of clinical immunity, one which reduces the risk of death from malaria and another which reduces the intensity of clinical symptoms. A third type of protective antimalarial immunity is antiparasitic immunity, which directly reduces the number of parasites in an infected individual. These are epidemiological definitions of immunity. The actual cellular and molecular mechanisms of immunity may overlap to a considerable degree between these categories. The number of malarial inoculations experienced, and the intervals between them, are all-important to the malaria immune status of an individual. In the case of acute attacks of Plasmodium falciparum malaria, it is possible that a degree of immunity to some

*Corresponding author. Email: jytmugisha@math.mak.ac.ug

Computational and Mathematical Methods in Medicine 
aspects of severe, life-threatening disease may be achieved after only one or two infections [10]. It is noted that a single untreated attack of $P$. falciparum malaria in a non immune individual carries a risk of death that may be anywhere from a few percent to at least $20-30 \%$ according to circumstances [1].

Incidence of malaria disease tends to decline uniformly with age such that children living in areas of high endemicity experience less frequent episodes of malaria after the age of 5 years [20].

People living in regions where malaria is endemic develop an acquired immunity to malaria which enables them to remain asymptomatic while still carrying parasites. The acquired partial immunity to the disease reduces the frequency of clinical attacks, although it does not necessarily reduce parasite prevalence. It is not sterile and requires continual boosting. This process is slow and may take years or decades to develop [13]. However, low level exposure to infection is important and acts as vaccination and develops immunity against the disease [9]. Thus, humans are susceptible to re-infections because the immune protection may wane over time (temporary immunity) or may not be fully protective (partial immunity). Dietz et al. [6] note that in some cases a partial cure may be worse than none since the reduction in immunological function due to short-term suppression of the disease may later result in higher levels of morbidity.

Malaria endemicity may be categorized into three types, according to MacDonald [19]. One is stable endemic malaria. Stable malaria occurs when a population is continuously exposed to a fairly constant rate of malarial inoculation (sub Saharan Africa). The next category is unstable endemic malaria, under which a population is subjected to more or less permanent malaria transmission (Asia, Latin America, Carribean, Mediterranean) but under circumstances in which there are large fluctuations in the rates at which malarial inoculations are delivered to individuals within the population. In terms of disease and immunity, these fluctuations become especially significant when they cause individuals to experience intervals of a year to several years between inoculations of malaria. The third type is epidemic malaria. This is, in effect, an extreme form of unstable malaria. It occurs when a population, or even a small group of individuals, is subjected to an increase in malaria transmission rates above that previously or normally experienced (highland Africa, Latin America).

There are at least 300 million acute cases of malaria each year globally, resulting in more than a million deaths. Around $90 \%$ of these deaths occur in Africa, mostly in young children. Malaria is Africa's leading cause of under-five mortality (20\%) and constitutes $10 \%$ of the continent's overall disease burden [22]. The climatic conditions in Africa are highly conducive to malaria transmission, being warm and humid with relatively few fluctuations. This supports longevity of the vector mosquitoes and rapid development of the parasites within them. All of these features combine to a recipe for stable and, indeed, generally intense malaria transmission.

Many malaria models have been used $[2,6,15,17,21]$ to study the dynamics of malaria at population level. Some of these have been used to consider the effect of different strategies such as vector control and use of vaccines on the transmission dynamics of malaria. The effect of acquired immunity was proposed by Dietz et al. [6] in the Garki model. This is was extended by Bailey [4] and Aron [3] .

In this paper, we extend these models to investigate the patterns of malaria transmission in the presence of vital (birth and death) dynamics both in the human and mosquito populations, which are usually ignored with a general reason that the life expectancy of mosquito vector is normally too short to affect the pattern. The assumptions of equal birth and death rates in all 
human epidemiological classes as in Koella and Antia [15] seems to be unrealistic in most malaria endemic regions. We also incorporate the role of acquired immunity and the rate of recovery of the infected humans because these are vital in the sub Saharan setting where we have continuous exposure to re-infection. Unlike in Koella and Antia [15] and Koella and Boate [16], where they ignore disease induced mortality, our model takes this into account since malaria is a major killer disease in Africa. The model is built on the system of equations and assumptions similar to those in Tumwiine et al. [24], but with interest in finding out the global behavioural patterns of both human and vector populations at endemic levels, under the influence of acquired immunity and effective recovery of the infected human hosts.

\section{Model description and formulation}

As in Tumwiine et al. [24], the human population is divided into three epidemiological classes that include the susceptible class $S_{\mathrm{H}}$, infective class $I_{\mathrm{H}}$ and immune class $R_{\mathrm{H}}$. The mosquito population is divided into two epidemiological classes, the susceptible class $S_{\mathrm{V}}$, and infective class $I_{\mathrm{V}}$. The vector population does not include immune class [4,12] as mosquitoes never recover from infection; that is, their infective period ends with their death due to their relatively short life-cycle. There is no vertical transmission and all the newborns are susceptible with a per capita birth rate $\lambda_{h}$. The human immune individuals lose their immunity at a constant rate $\gamma$, and the infected human individuals recover at a constant rate $\nu$ to join the susceptible. The infected individuals acquire immunity at constant rate $r$ and may die due to the disease at a rate $\delta$. The natural per capita death rate is assumed to be the same constant $\mu_{\boldsymbol{h}}$ for all humans. The mosquito population has $\lambda_{v}$ and $\mu_{v}$ as the natural per capita birth and mortality rates respectively. The infected female mosquitoes bite humans at a rate $a$. The fraction of the bites that successfully infect humans is $b$ and $c$ is the fraction of bites that infect mosquitoes when they bite infected humans. The incidence term is of the standard form with the terms $a b S_{\mathrm{H}} I_{\mathrm{V}} / N_{\mathrm{H}}$ denoting the rate at which the human hosts $S_{\mathrm{H}}$ get infected by infected mosquitoes $I_{\mathrm{V}}$ and $a c S_{\mathrm{V}} I_{\mathrm{H}} / N_{\mathrm{H}}$ for the rate at which the susceptible mosquitoes $S_{\mathrm{V}}$ are infected by the infected human hosts $I_{\mathrm{H}}$. The rate of infection of human host $S_{\mathrm{H}}$ by infected vector $I_{\mathrm{V}}$ is dependent on the total number of humans $N_{\mathrm{H}}$ available per infected vector [21]. The above description leads to the compartmental diagram in figure 1 . From the

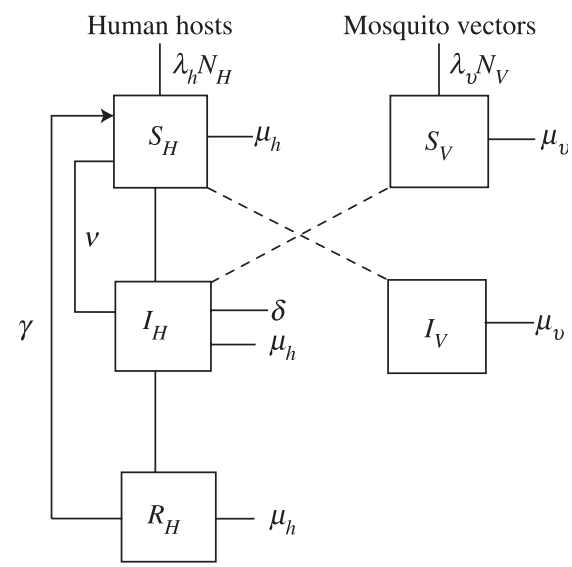

Figure 1. The host-vector dynamics of malaria transmission with temporary immunity. 
compartmental diagram in figure 1 , we have the following set of equations for the dynamics of the model:

$$
\begin{gathered}
\frac{\mathrm{d} S_{\mathrm{H}}}{\mathrm{d} t}=\lambda_{h} N_{\mathrm{H}}-\frac{a b S_{\mathrm{H}} I_{\mathrm{V}}}{N_{\mathrm{H}}}+\nu I_{\mathrm{H}}+\gamma R_{\mathrm{H}}-\mu_{h} S_{\mathrm{H}} \\
\frac{\mathrm{d} I_{\mathrm{H}}}{\mathrm{d} t}=\frac{a b S_{\mathrm{H}} I_{\mathrm{V}}}{N_{\mathrm{H}}}-\nu I_{\mathrm{H}}-r I_{\mathrm{H}}-\delta I_{\mathrm{H}}-\mu_{h} I_{\mathrm{H}} \\
\frac{\mathrm{d} R_{\mathrm{H}}}{\mathrm{d} t}=r I_{\mathrm{H}}-\gamma R_{\mathrm{H}}-\mu_{h} R_{\mathrm{H}} \\
\frac{\mathrm{d} S_{\mathrm{V}}}{\mathrm{d} t}=\lambda_{v} N_{\mathrm{V}}-\frac{a c S_{\mathrm{V}} I_{\mathrm{H}}}{N_{\mathrm{H}}}-\mu_{v} S_{\mathrm{V}} \\
\frac{\mathrm{d} I_{\mathrm{V}}}{\mathrm{d} t}=\frac{a c S_{\mathrm{V}} I_{\mathrm{H}}}{N_{\mathrm{H}}}-\mu_{v} I_{\mathrm{V}}
\end{gathered}
$$

with the total population sizes $S_{\mathrm{H}}+I_{\mathrm{H}}+R_{\mathrm{H}}=N_{\mathrm{H}}$ and $S_{\mathrm{V}}+I_{\mathrm{V}}=N_{\mathrm{V}}$.

\section{Model analysis}

In this section we carry out qualitative analysis of the model to investigate stability of the steady states. But first, we transform the system of populations into a system of proportions. The equations are obtained by differentiating each proportion with respect to time $t$. The proportions for the system are $s_{h}=S_{\mathrm{H}} / N_{\mathrm{H}}, i_{h}=I_{\mathrm{H}} / N_{\mathrm{H}}, r_{h}=R_{\mathrm{H}} / N_{\mathrm{H}}, s_{v}=S_{\mathrm{V}} / N_{\mathrm{V}}$ and $i_{v}=I_{\mathrm{V}} / N_{\mathrm{V}}$ in the classes $S_{\mathrm{H}}, I_{\mathrm{H}}, R_{\mathrm{H}}, S_{\mathrm{V}}$ and $I_{\mathrm{V}}$ of the populations respectively and $m=N_{\mathrm{V}} / N_{\mathrm{H}}$ is the female vector-host ratio, defined as the number of female mosquitoes per human host $[2,7,23]$. This gives the following system of equations:

$$
\begin{gathered}
\frac{\mathrm{d} s_{h}}{\mathrm{~d} t}=\lambda_{h}\left(1-s_{h}\right)-a b m s_{h} i_{v}+\nu i_{h}+\gamma r_{h}+\delta s_{h} i_{h} \\
\frac{\mathrm{d} i_{h}}{\mathrm{~d} t}=a b m s_{h} i_{v}-\left(\nu+r+\lambda_{h}+\delta\right) i_{h}+\delta i_{h}^{2} \\
\frac{\mathrm{d} r_{h}}{\mathrm{~d} t}=r i_{h}-\left(\gamma+\lambda_{h}\right) r_{h}+\delta i_{h} r_{h} \\
\frac{\mathrm{d} s_{v}}{\mathrm{~d} t}=\lambda_{v}\left(1-s_{v}\right)-a c i_{h} s_{v}
\end{gathered}
$$




$$
\frac{\mathrm{d} i_{v}}{\mathrm{~d} t}=a c s_{v} i_{h}-\lambda_{v} i_{v}
$$

with the restrictions $S_{h}+i_{h}+r_{h}=1$ and $S_{v}+i_{v}=1$.

The equilibria are obtained by equating the right-hand side of system (6)-(10) to zero. Thus,

$$
\begin{gathered}
\lambda_{h}\left(1-s_{h}\right)-a b m s_{h} i_{v}+\nu i_{h}+\gamma r_{h}+\delta s_{h} i_{h}=0 \\
a b m s_{h} i_{v}-\left(\nu+r+\lambda_{h}+\delta\right) i_{h}+\delta i_{h}^{2}=0 \\
r i_{h}-\left(\gamma+\lambda_{h}\right) r_{h}+\delta i_{h} r_{h}=0 \\
\lambda_{v}\left(1-s_{v}\right)-a c i_{h} s_{v}=0 \\
a c s_{v} i_{h}-\lambda_{v} i_{v}=0
\end{gathered}
$$

We express the solutions in terms of $i_{h}$ for easy analysis of the steady states to obtain

$$
\left.\begin{array}{rl}
s_{h} & =\frac{\left[\lambda_{h}+\gamma+(\nu-\gamma) i_{h}\right]\left[\lambda_{v}+a c i_{h}\right]}{\left(\lambda_{h}+\gamma-\delta i_{h}\right)\left(\lambda_{v}+a c i_{h}\right)+a^{2} b m c i_{h}} \\
r_{h} & =\frac{r i_{h}}{\lambda_{h}+\gamma-\delta i_{h}} \\
s_{v} & =\frac{\lambda_{v}}{\lambda_{v}+a c i_{h}} \\
i_{v} & =\frac{a c i_{h}}{\lambda_{v}+a c i_{h}}
\end{array}\right\}
$$

\subsection{Disease-free equilibrium $E_{0}$}

At the disease-free equilibrium, we have $i_{h}=i_{v}=0$ and the equations give the steady state, $E_{0}=(1,0,0,1,0)$. The local stability of this point is established from the Jacobian of the system (6)-(10) evaluated at $E_{0}$. The Jacobian matrix of the system (6)-(10) is given by

$$
J_{E}=\left[\begin{array}{ccccc}
-\left(\lambda_{h}+a b m i_{v}-\delta i_{h}\right) & \nu+\delta s_{h} & \gamma & 0 & -a b m s_{h} \\
a b m i_{v} & -\left(\nu+r+\lambda_{h}+\delta\right)+2 \delta i_{h} & 0 & 0 & a b m s_{h} \\
0 & r+\delta r_{h} & \delta i_{h}-\left(\lambda_{h}+\gamma\right) & 0 & 0 \\
0 & -a c s_{v} & 0 & -\left(\lambda_{v}+a c i_{h}\right) & 0 \\
0 & a c s_{v} & 0 & a c i_{h} & -\lambda_{v}
\end{array}\right]
$$


The Jacobian matrix (17) evaluated at $E_{0}=(1,0,0,1,0)$ gives

$$
J_{E_{0}}=\left[\begin{array}{ccccc}
-\lambda_{h} & \nu+\delta & \gamma & 0 & -a b m \\
0 & -\left(\nu+r+\lambda_{h}+\delta\right) & 0 & 0 & a b m \\
0 & r & -\left(\lambda_{h}+\gamma\right) & 0 & 0 \\
0 & -a c & 0 & -\lambda_{v} & 0 \\
0 & a c & 0 & 0 & -\lambda_{v}
\end{array}\right]
$$

We observe that matrix (18) has negative eigenvalue $-\lambda_{h},-\left(\lambda_{h}+\gamma\right)$ and $-\lambda_{v}$, and the remaining two can be obtained from the $2 \times 2$ block matrix given by

$$
A=\left[\begin{array}{cc}
-\left(\nu+r+\lambda_{h}+\delta\right) & a b m \\
a c & -\lambda_{v}
\end{array}\right]
$$

whose trace and determinant are given by

$$
\begin{gathered}
\operatorname{Tr} A=-\left(\lambda_{v}+\lambda_{h}+\nu+r+\delta\right)<0 \\
\operatorname{Det} A=\lambda_{v}\left(\nu+r+\lambda_{h}+\delta\right)\left(1-R_{0}\right)>0 \text { if } R_{0}<1,
\end{gathered}
$$

where

$$
R_{0}=\frac{a^{2} b m c}{\lambda_{v}\left(\nu+r+\lambda_{h}+\delta\right)},
$$

Thus, $E_{0}$ is locally asymptotically stable if and only if $R_{0}<1$, and we have thus established the following Lemma:

Lemma 1 . The disease-free equilibrium $E_{0}$ is locally stable if $R_{0}<1$ and unstable if $R_{0}>1$.

The quantity $R_{0}$ is the basic reproduction number of the disease. It represents the average number of new infections produced by one infected individual introduced in an otherwise susceptible population. It is a useful quantity in the study of a disease as it sets the threshold for its establishment. If $R_{0}<1$, then the disease-free equilibrium is locally stable.

\subsection{Global stability of disease-free equilibrium $E_{0}$}

As in Tumwiine et al. [24], we use the following theorem to prove the global stability of the disease-free equilibrium point:

THEOREM 1. The disease-free equilibrium $E_{0}=(1,0,0,1,0)$ of (6)-(10) is globally asymptotically stable if $R_{0} \leq 1$ and unstable if $R_{0}>1$. 
Proof. Consider the following Lyapunov function, $L=a c i_{h}+\left(\nu+r+\lambda_{h}+\delta\right) i_{v}$. Then

$$
\begin{aligned}
L^{\prime} & =a^{2} b m c s_{h} i_{v}-a c i_{h}\left[\nu+r+\lambda_{h}+\delta-\delta i_{h}\right]+\left(\nu+r+\lambda_{h}+\delta\right)\left[a c i_{h} s_{v}-\lambda_{v} i_{v}\right] \\
& =a^{2} b m c s_{h} i_{v}-\lambda_{v} i_{v}\left(\nu+r+\lambda_{h}+\delta\right)+a c i_{h}\left[\delta i_{h}+\left(\nu+r+\lambda_{h}+\delta\right)\left(s_{v}-1\right)\right] \\
& =\left(\nu+r+\lambda_{h}+\delta\right) \lambda_{v} i_{v}\left[\frac{a^{2} b m c s_{h}}{\lambda_{v}\left(\nu+r+\lambda_{h}+\delta\right)}-1\right]+a c i_{h}\left[\delta i_{h}-\left(\nu+r+\lambda_{h}+\delta\right) i_{v}\right] \\
& =\lambda_{v} i_{v}\left(\nu+r+\lambda_{h}+\delta\right)\left[R_{0} s_{h}-1\right]-a c i_{h}\left[\left(\nu+r+\lambda_{h}+\delta\right) i_{v}-\delta i_{h}\right] \\
& \leq \lambda_{v} i_{v}\left(\nu+r+\lambda_{h}+\delta\right)\left[R_{0} s_{h}-1\right] \quad \leq 0 \quad \text { if } \quad R_{0} \leq 1
\end{aligned}
$$

$L^{\prime}=0$ holds if and only if $R_{0}=1$ and $i_{h}=i_{v}=0$. Thus, by Lyapunov-Lasalle's Theorem [11], every solution that starts in the feasible region where the solutions have biological meaning approaches $E_{0}$ as $t \rightarrow+\infty$. This shows that the disease eventually disappears from the community. Hence, the disease-free equilibrium point is globally asymptotically stable and hence Theorem 1 is proved.

The reproduction number depends on the product of the transmission coefficients, $a b m$ and $a c$, the average residence time, $1 /\left(\nu+r+\lambda_{h}+\delta\right)$ in the infective class and the average life span, $1 / \lambda_{v}$ of the mosquito. It is also dependent on the rate of acquisition of immunity, $r$ rate of recovery from infection, $\nu$ and disease induced mortality rate, $\delta$.

We can quantify that higher values of coefficients of transmission between humans and mosquito vectors $m a, b$ and $c$ can allow the establishment of the disease. There is need to pay attention to processes that can limit the spread of the disease such as protection of the vulnerable groups from human-mosquito interaction by use of treated mosquito nets, providing prompt and effective treatment to those who are sick.

Based on the expression for, $R_{0}$ we observe that lowering the average residence time in the infectious class is essential in the fight against the outbreaks. This can be done for example through epidemiological parameters such as seeking prompt and appropriate treatment $\delta$, prompt and appropriate treatment, $\nu$, and increased continued exposure that guarantee $R_{0}<1$.

\subsection{Local stability of endemic equilibrium $E_{1}$}

Let $r_{h}=1-S_{h}-i_{h}$ and $S_{v}=1-i_{v}$, then we can reduce system (6)-(10) to a 3-dimensional system whose Jacobian becomes

$$
J_{E_{1}}=\left[\begin{array}{ccc}
-\left(\lambda_{h}+\gamma+a b m i_{v}-\delta i_{h}\right) & \nu+\delta s_{h}-\gamma & -a b m s_{h} \\
a b m i_{v} & -\left(\nu+r+\lambda_{h}+\delta\right)+2 \delta i_{h} & a b m s_{h} \\
0 & a c\left(1-i_{v}\right) & -\lambda_{v}-a c i_{h}
\end{array}\right]
$$

The expressions for solutions in equations (16) should satisfy conditions $\lambda_{h}+$ $\gamma_{-\delta i h}>0$ for the endemic equilibrium $E 1=\left(S_{h}, i_{h}, i_{v}\right)>0$ to exist. The characteristic equation of $J_{E_{1}}$ is

$$
\xi^{3}+A_{1} \xi^{2}+A_{2} \xi+A_{3}=0
$$


where, $\xi$ is the eigenvalue and $A_{1}, A_{2}$ and $A_{3}$ are constants given by the expressions

$$
\begin{aligned}
& A_{1}=\lambda_{v}+a c i_{h}+\lambda_{h}+\gamma+a b m i_{v}-\delta i_{h}+\nu+r+\lambda_{h}+\delta-2 \delta i_{h} \\
& \leq \lambda_{v}+a c i_{h}+\lambda_{h}+\gamma+a b m i_{v}-\delta i_{h}+\nu+r+\lambda_{h}+\delta-\delta i_{h} \\
& A_{2}=\left(\lambda_{v}+a c i_{h}\right)\left(\nu+r+\lambda_{h}+\delta-2 \delta i_{h}\right)-a^{2} b m c\left(1-i_{v}\right) s_{h} \\
& +\left(\lambda_{h}+\gamma+a b m i_{v}-\delta i_{h}\right)\left(\lambda_{v}+a c i_{h}+\nu+r+\lambda_{h}+\delta-2 \delta i_{h}\right)+\left(\gamma-\nu-\delta s_{h}\right) a b m i_{v} \\
& \leq\left(\lambda_{v}+a c i_{h}\right)\left(\nu+r+\lambda_{h}+\delta-\delta i_{h}\right)-\lambda_{v}\left(\nu+r+\lambda_{h}+\delta-\delta i_{h}\right) \\
& +\left(\lambda_{h}+\gamma+a b m i_{v}-\delta i_{h}\right)\left(\lambda_{v}+a c i_{h}+\nu+r+\lambda_{h}+\delta-\delta i_{h}\right) \\
& +\frac{a b m i_{v}\left[\left(\lambda_{h}+\gamma\right)\left(1-s_{h}\right)-\left(\nu+r+\lambda_{h}+\delta-\delta i_{h}\right) i_{h}\right]}{i_{h}} \\
& =\operatorname{aci}_{h}\left(\nu+r+\lambda_{h}+\delta-\delta i_{h}\right)+\left(\lambda_{v}+a c i_{h}\right)\left(\lambda_{h}+a b m i_{v}+\gamma-\delta i_{h}\right) \\
& +\left(\lambda_{h}+\gamma-\delta i_{h}\right)\left(\nu+r+\lambda_{h}+\delta-\delta i_{h}\right)+\frac{a b m i_{v}\left[\left(\lambda_{h}+\gamma\right)\left(1-s_{h}\right)\right]}{i_{h}} \\
& A_{3}=\left(\lambda_{h}+\gamma+a b m i_{v}-\delta i_{h}\right)\left[\left(\lambda_{v}+a c i_{h}\right)\left(\nu+r+\lambda_{h}+\delta-2 \delta i_{h}\right)-a^{2} b m c\left(1-i_{v}\right) s_{h}\right] \\
& +a^{3} b^{2} m^{2} c s_{h}\left(1-i_{v}\right)+\left(\gamma-\nu-\delta s_{h}\right)\left(\lambda_{v}+a c i_{h}\right) a b m i_{v} \\
& \leq\left(\lambda_{h}+\gamma+a b m i_{v}-\delta i_{h}\right)\left[\left(\lambda_{v}+a c i_{h}\right)\left(\nu+r+\lambda_{h}+\delta-\delta i_{h}\right)\right. \\
& \left.-\lambda_{v}\left(\nu+r+\lambda_{h}+\delta-\delta i_{h}\right)\right] \\
& +a b m \lambda_{v}\left(\nu+r+\lambda_{h}+\delta-\delta i_{h}\right)+\left(\gamma-\nu-\delta s_{h}\right)\left(\lambda_{v}+a c i_{h}\right) a b m i_{v} \\
& =a c i_{h}\left(\lambda_{h}+\gamma+a b m i_{v}-\delta i_{h}\right)\left(\nu+r+\lambda_{h}+\delta-\delta i_{h}\right)+a b m \lambda_{v}\left(\nu+r+\lambda_{h}+\delta-\delta i_{h}\right) \\
& +\frac{\left(\lambda_{v}+a c i_{h}\right) a b m i_{v}\left[\left(\lambda_{h}+\gamma\right)\left(1-s_{h}\right)-\left(\nu+r+\lambda_{h}+\delta-\delta i_{h}\right) i_{h}\right]}{i_{h}} \\
& =a c i_{h}\left(\lambda_{h}+\gamma-\delta i_{h}\right)\left(\nu+r+\lambda_{h}+\delta-\delta i_{h}\right)+a b m \lambda_{v}\left(\nu+r+\lambda_{h}+\delta-\delta i_{h}\right)\left(1-i_{v}\right) \\
& +\frac{\left(\lambda_{v}+a c i_{h}\right) a b m i_{v}\left(\lambda_{h}+\gamma\right)\left(1-s_{h}\right)}{i_{h}}
\end{aligned}
$$

then

$$
\begin{aligned}
A_{1} A_{2}-A_{3}= & \left(\lambda_{v}+a c i_{h}\right)\left[a c i_{h}\left(\nu+r+\lambda_{h}+\delta-\delta i_{h}\right)+\left(\lambda_{v}+a c i_{h}\right)\left(\lambda_{h}+\gamma+a b m i_{v}-\delta i_{h}\right)\right] \\
& +\left(\lambda_{h}+\gamma+a b m i_{v}-\delta i_{h}\right)\left(\nu+r+\lambda_{h}+\delta-\delta i_{h}\right)\left[a c i_{h}\left(\nu+r+\lambda_{h}+\delta-\delta i_{h}\right)\right. \\
& +\left(\lambda_{v}+a c i_{h}\right)\left(\lambda_{h}+a b m i_{v}+\gamma-\delta i_{h}\right)+\left(\lambda_{h}+\gamma-\delta i_{h}\right)\left(\nu+r+\lambda_{h}+\delta-\delta i_{h}\right) \\
& \left.+\frac{a b m i_{v}\left(\lambda_{h}+\gamma\right)\left(1-s_{h}\right)}{i_{h}}\right]+\lambda_{v}\left(\lambda_{h}+\gamma-\delta i_{h}\right)\left(\nu+r+\lambda_{h}+\delta-\delta i_{h}\right) \\
& +a b m \lambda_{v}\left(\nu+r+\lambda_{h}+\delta-\delta i_{h}\right)\left(i_{v}-1\right)
\end{aligned}
$$

By the fact that $\lambda_{h}+\gamma-\delta i_{h}>0$, which follows from the equilibrium solutions given in equation (16), we deduce that $A_{1}>0, A_{2}>0, A_{3}>0$ and $A_{1} A_{2}-A_{3}>0$. Hence by the Routh-Hurwitz criteria, the endemic equilibrium is locally stable.

For global stability of the endemic equilibrium, we use the numerical analysis in the following section to predict the long term pattern of both the host and vector populations. 
Table 1. The model parameter estimates.

\begin{tabular}{|c|c|c|}
\hline Parameter & Value & Reference \\
\hline$a$, the average daily biting rate on man by a single mosquito & $0.29 /$ day & {$[14,18]$} \\
\hline$b$, the proportion of bites on man that produce an infection & 0.75 & {$[18]$} \\
\hline$c$, the probability that a mosquito becomes infectious & 0.75 & [18] \\
\hline$\gamma$, the per capita rate of loss of immunity in human hosts & $0.000017 /$ day & [14] \\
\hline$r$, the rate at which human hosts acquire immunity & $0.00019 /$ day & [5] \\
\hline$\delta$, the per capita death rate due to the disease & 0.333 & {$[25]$} \\
\hline$\nu$, the rate of recovery of human hosts from the disease & $0.0022 /$ day & {$[3,8]$} \\
\hline$\lambda_{h}$, the per capita natural birth rate of humans & $0.0015875 /$ day & [8] \\
\hline$\lambda_{v}$, the per capita natural birth rate of the mosquitoes & $0.071 /$ day & [8] \\
\hline$\mu_{h}$, the per capita natural death rate of the humans & $0.00004 /$ day & [5] \\
\hline$\mu_{v}$, the per capita natural death rate of the mosquitoes & $0.05 /$ day & [19] \\
\hline
\end{tabular}

\subsection{Numerical analysis of the model}

In this section, we present the numerical analysis of the model. The parameter values in table 1 are used in the simulations to illustrate the behaviour of the model. In figures 2 and 3, we observe that in the early stages of the epidemic, there is a high prevalence of malaria because of a large proportion of infected mosquito vectors that results in a significant decrease in the number of susceptible human hosts. As the proportions of infected humans and infected mosquitoes decrease and remain at low level values, we observe a dramatic increase in the immune class. Since the acquired immunity to malaria develops gradually due to continuous exposure to infections and can be lost, the proportion of the immune individuals declines as they join the susceptible class. In the absence of infected human hosts and mosquito vectors, the proportion of the immune class decreases as a result of immunity loss and this leads to an increase in the human susceptibles. We eventually have a higher proportion of immune humans (figure 2c) compared to the proportion of susceptible humans (figure 2a). There are damped oscillations of the proportions until an endemic equilibrium level is eventually reached and this converges to a steady state that is asymptotically stable. These numerical results support the results earlier obtained analytically that the endemic equilibrium is stable.

\section{Discussion}

We proposed a model with standard incidence for the dynamics of malaria within human hosts and mosquito vectors in which the reservoir of the susceptible human hosts is refilled by individuals who lose their immunity to the disease and newborns. The model was then reformulated in terms of the proportions of the classes of the respective populations. Model analysis and simulations were carried out. Two equilibria points were obtained and their stability analysed.

It was established that for the basic reproduction number, $R_{0} \leq 1$, the disease-free equilibrium is globally stable so that the disease always dies out, and if $R_{0}>1$, the diseasefree equilibrium point is unstable while the endemic equilibrium emerges as a unique equilibrium point, re-invasion is always possible and the disease never dies out.

Thus, a threshold population size is necessary for the perpetuation of the disease. These may be based on the parameters of the threshold quantity, $R_{0}$. We notice that in order to reduce the basic reproduction number below 1 , intervention strategies need to be focused on treatment and reduction on the contact between mosquito vector and human host. Thus, there 
(a)
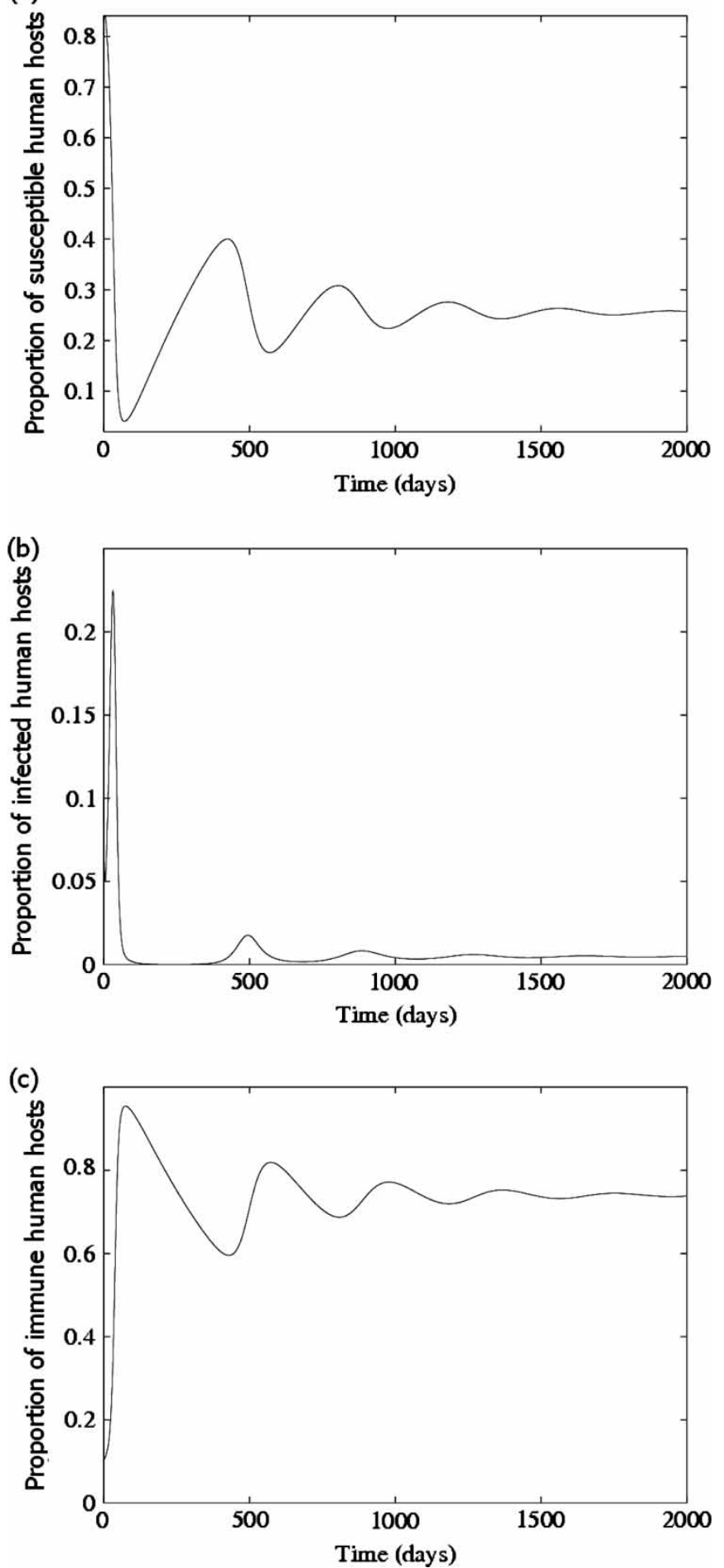

Figure 2. Endemic patterns of the susceptible, infected and immune human populations. 

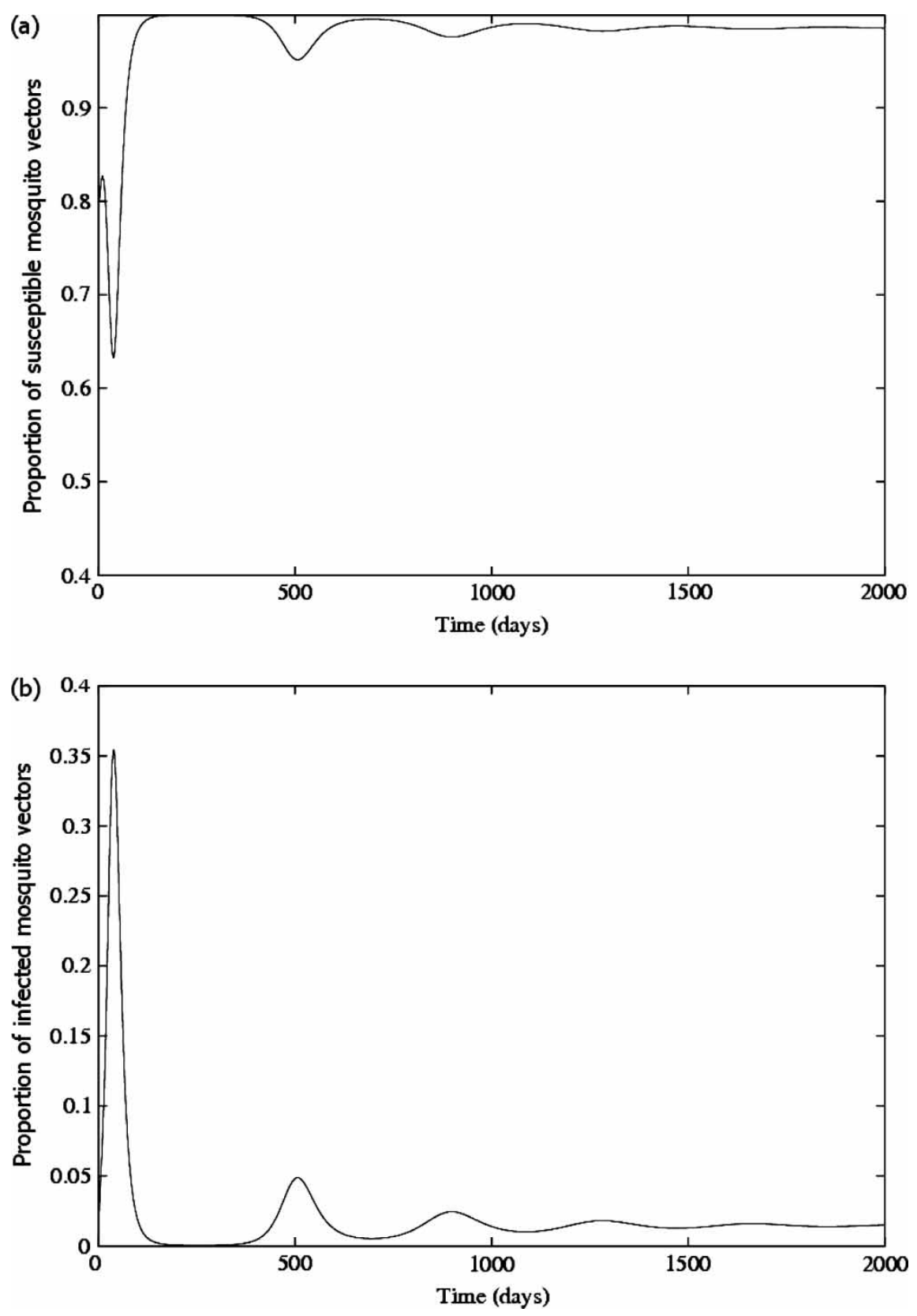

Figure 3. Endemic patterns of the susceptible and infected mosquito populations. 
is need for effective drugs, treated bed nets and insecticides that would reduce the mosquito population. Since malaria induced immunity is not everlasting, it remains a major obstacle to eradicate the disease even if individuals are protected.

Numerical analysis revealed that the endemic equilibrium converges to a steady state. From figures 2(b) and 3(b), we observe that there is a strong relationship between the proportion of infected mosquitoes and infected humans in the same locality in a way that a rise in the proportion of infected mosquitoes results in an increase in the proportion of infected humans. Therefore, control efforts aimed at lowering the infectivity of infected individuals to the mosquito vector will contribute greatly to the lowering of the malaria transmission and this will eventually lower the prevalence of malaria and the incidence of the disease in that locality. This can be achieved by prompt provision of effective antimalarial drugs to reduce transmission and morbidity. Thus, from the model, it is noted that recurrent and temporary immunity leads to oscillatory pattern in all the populations of the model.

\section{References}

[1] Alles, H.K., Mendis, K.N. and Carter, R., 1998, Malaria mortality rates in South Asia and in Africa: implications for malaria control, Parasitology Today, 14, 369-375.

[2] Aneke, S.J., 2002, Mathematical modelling of drug resistant malaria parasites and vector populations, Mathematical Methods in the Applied Sciences, 90, 385-396.

[3] Aron, J.L., 1988, Mathematical modelling of immunity to malaria, Mathematical Biosciences, 90, 385-396.

[4] Bailey, N.T.J., 1982, The Biomathematics of Malaria (London: Charles Griff).

[5] Coutinho, F.A.B., Burattini, M.N., Lopez, L.F. and Massad, E., 2005, An approximate threshold condition for non-autonomous system: an application to a vector-borne infection, Mathematics and Computers in Simulation, 70, $149-158$.

[6] Dietz, K., Molineaux, L. and Thomas, A., 1974, A malaria model tested in the African Savannah, Bulletin of the World Health Organization, 50, 347-357.

[7] Freeman, J., Laserson, K.F., Petralanda, I. and Spielman, A., 1999, Effect of chemotherapy on malaria transmission among Yonomami Amerindians: simulated consquences of placebo treatment, The American Journal of Tropical Medicine and Hygiene, 60(5), 774-780.

[8] Gemperli, A., Vounatsou, P., Sogoba, N. and Smith, T., 2006, Malaria mapping using transmission models: application to survey data, American Journal of Epidemiology, 163, 289-297.

[9] Ghosh, A.K., Chattopadhyay, J. and Tapaswi, P.K., 1996, Immunity boosted by low exposure to infection in an SIRS model, Ecological Modelling, 87, 227-233.

[10] Gupta, S., Snow, R.W., Donnelly, C.A., Marsh, K. and Newbold, C., 1999, Immunity to non-cerebral severe malaria is acquired after one or two infections, Nature Medicine, 5, 340-343.

[11] Hale, J.K., 1969, Ordinary Differential Equations (New York: John Wiley).

[12] Hethcote, H.W., 1976, Qualitative analysis of communicable disease models, Mathematical Biosciences, 28, 335-356.

[13] Hviid, P., 2005, Natural acquired immunity to Plasmodium falciparum malaria in Africa, Acta Tropica, 95, 265-269.

[14] Ishikawa, H., Ishii, A., Nagai, N., Ohmae, H., Masakazu, H., Suguri, S. and Leafasia, J., 2003, A mathematical model for the transmission of Plasmodium vivax malaria, Parasitology International, 52, 81-93.

[15] Koella, J.C. and Antia, R., 2003, Epidemiological models for the spread of anti-malarial resistance, Malaria Journal, 2, 1-11.

[16] Koella, J.C. and Boate, C., 2003, A model for the co-evolution of immunity and immune evasion in vectorborne disease with implications for the epidemiology of malaria, The American Naturalist, 161, 698-707.

[17] Koella, J.C., 1991, On the use of mathematical models of malaria transmission, Acta Tropica, 49, 1-25.

[18] Laxminarayan, R., 2004, Act now or later? Economics of malaria resistance, The American Journal of Tropical Medicine and Hygiene, 71(2 suppl), 187-195.

[19] Macdonald, G., 1957, The Epidemiology and Control of Malaria (Oxford: Oxford University Press).

[20] Marsh, K., Otoo, L. and Hayes, R.H., 1989, Antibodies to blood stages antigens of Plasmodium falciparum in rural Gambians and their relationship to protection against infection, Transactions of the Royal Society of Tropical Medicine and Hygiene, 83, 293-303.

[21] Ngwa, G.A. and Shu, W.S., 2000, A mathematical model for endemic malaria with variable human and mosquito populations, Mathematics and Computer Modelling, 32, 747-763. 
[22] Olumese, P., 2005, Epidemiology and surveillance: changing the global picture of malaria-myth or reality?, Acta Tropica, 95, 265-269.

[23] Rogers, D.J., Randolph, S.E., Snow, R.W. and Hay, S.I., 2002, Satellite imagery in the study and forecast of malaria, Nature, 415, 710-715.

[24] Tumwiine, J., Mugisha, J.Y.T. and Luboobi, L.S., 2007, A mathematical model for the dynamics of malaria in a human host and mosquito vector with temporary immunity, Applied Mathematics and Computation, 189, $1953-1965$.

[25] Tumwiine, J., Luboobi, L.S. and Mugisha, J.Y.T., 2005, Modelling the effect of treatment and mosquitoes control on malaria transmission, International Journal of Management and Systems, 21(2), $107-124$. 


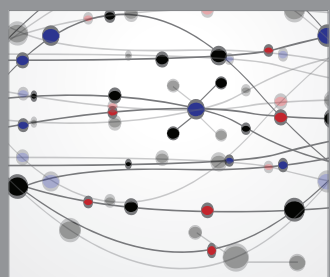

The Scientific World Journal
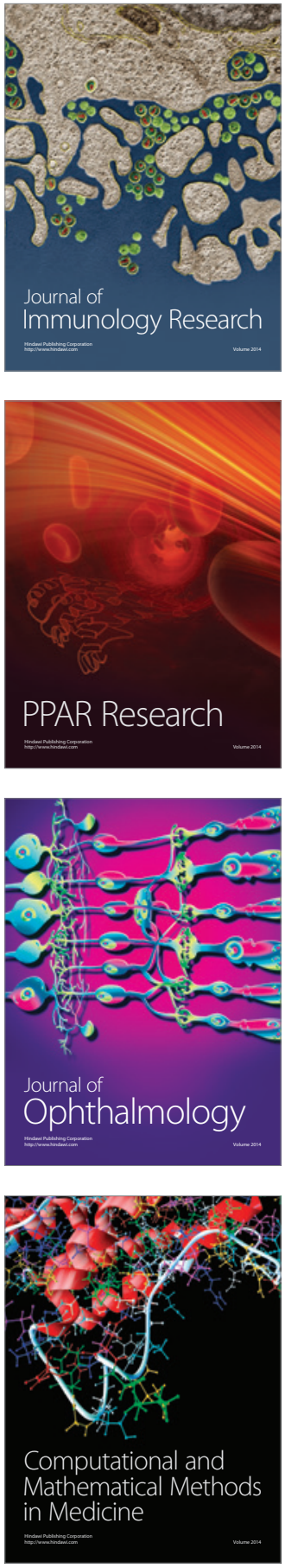

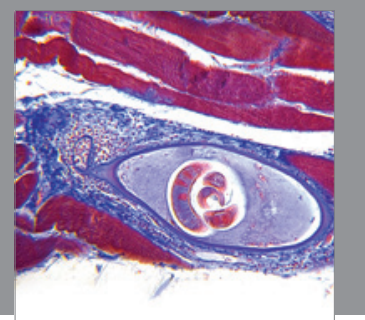

Gastroenterology

Research and Practice
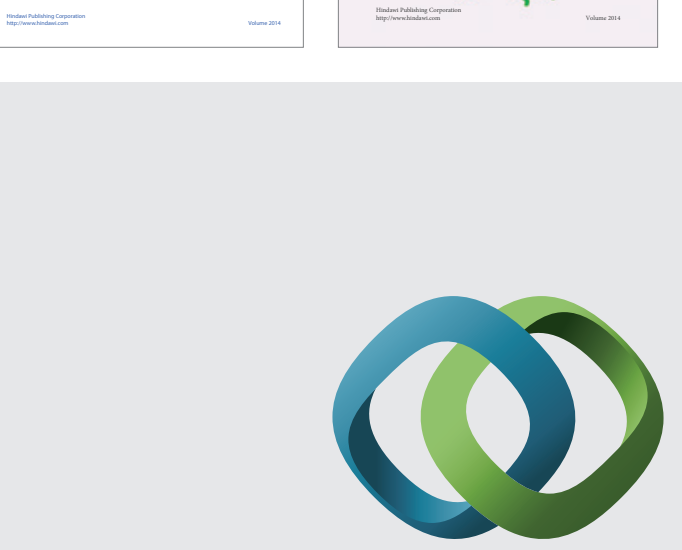

\section{Hindawi}

Submit your manuscripts at

http://www.hindawi.com
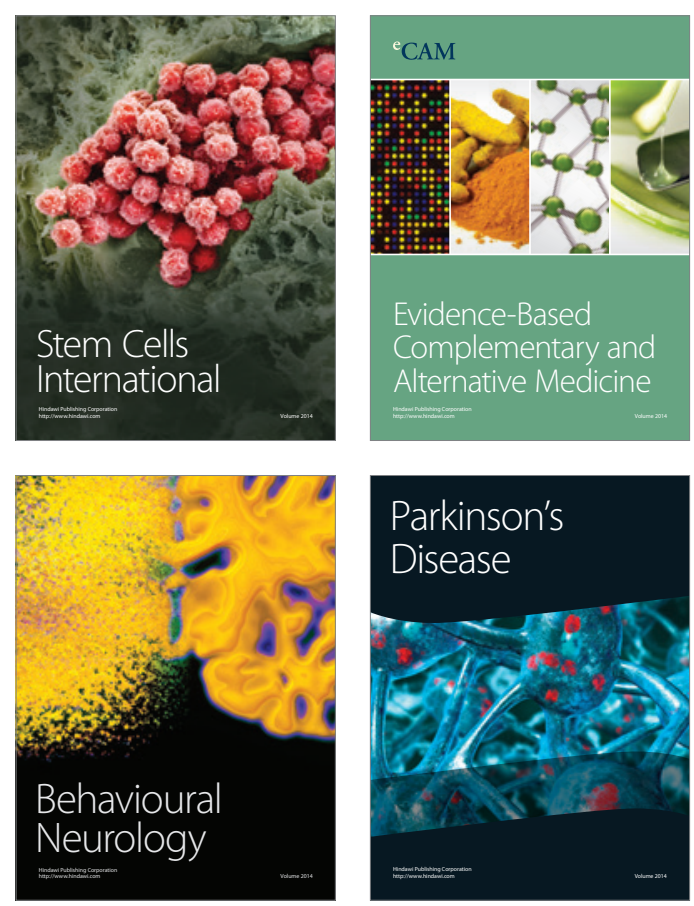

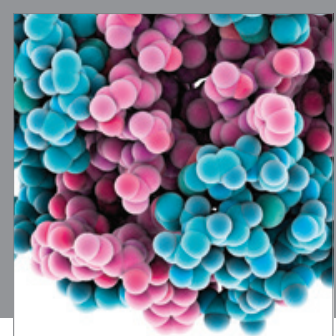

Journal of
Diabetes Research

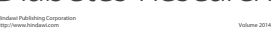

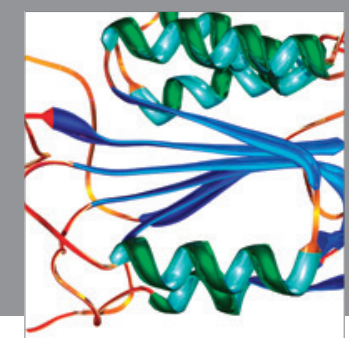

Disease Markers
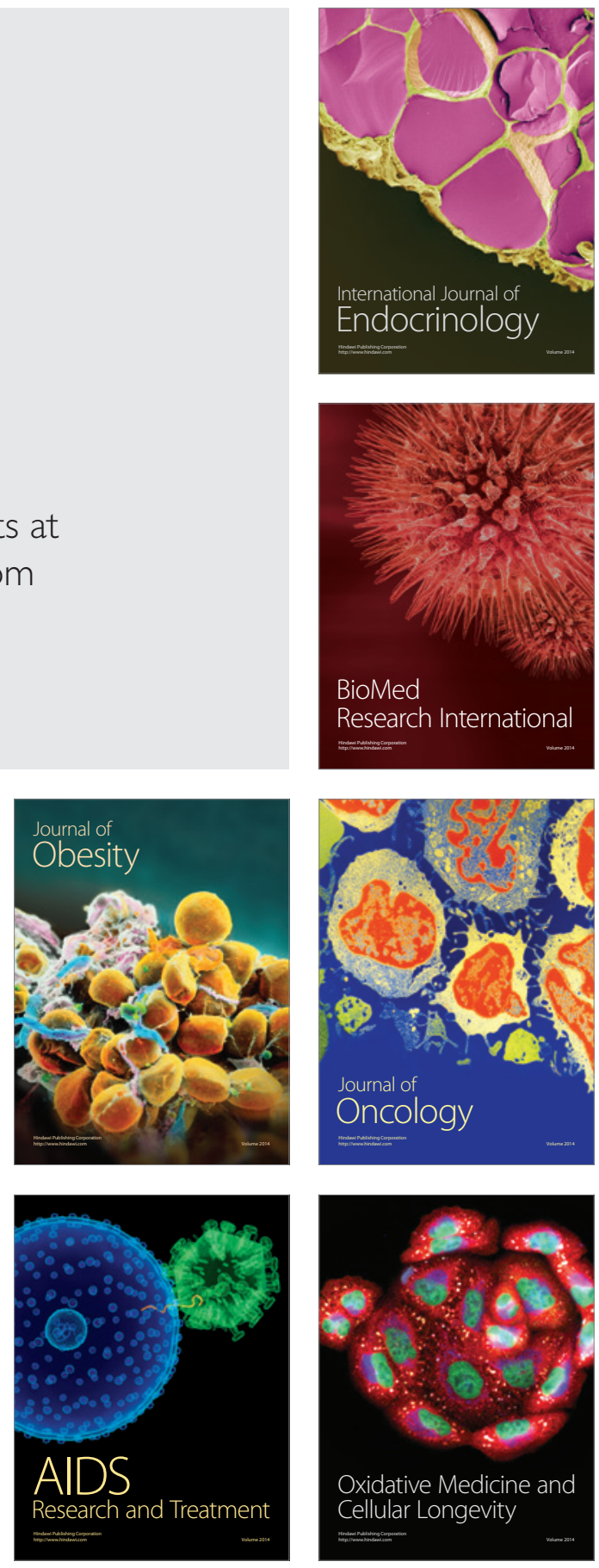